\title{
锐钛矿光催化降解苯酚：氟离子吸附的影响大于磷酸根
}

\author{
刘胜伟赵建军许宜铭* \\ (浙江大学化学系 硅材料国家重点实验室 杭州 310027)
}

\begin{abstract}
摘要 已知氟离子和磷酸根能加快锐钛矿和 $\mathrm{P} 25 \mathrm{TiO}_{2}$ 光催化降解苯酚等有机物, 但作用机理仍存在争议. 合成了不含 无机阴离子的锐钛矿, 并在其表面沉积 $0.52 \mathrm{wt} \% \mathrm{Pt}\left(\mathrm{Pt} / \mathrm{TiO}_{2}\right)$. 在初始 $\mathrm{pH} 5.2$ 的水溶液中, $99 \%$ 的氟离子和磷酸根分别为 $\mathrm{F}^{-}$和 $\mathrm{H}_{2} \mathrm{PO}_{4}^{-}$. 加入 $0.1 \sim 30 \mathrm{mmol} / \mathrm{L}$ 阴离子, 苯酚的光催化降解速率常数 $\left(k_{\mathrm{obs}}\right)$ 都增大, 证实了氟离子和磷酸根均具有正 效应. 有趣的是, $k_{\mathrm{obs}}$ 增加倍数均与阴离子吸附量呈线性关系, 其中氟离子的斜率大于磷酸根, 而 $\mathrm{Pt} / \mathrm{TiO}_{2}$ 的斜率大于 $\mathrm{TiO}_{2}$. 这表明阴离子的正效应源于吸附于固体表面的阴离子, 并且氟离子的活性大于磷酸根. (光)电化学测试表明, 氟 离子和磷酸根分别抑制和促进 $\mathrm{O}_{2}$ 还原, 但它们都促进苯酚氧化. 此外, 氟离子和磷酸根分别使 $\mathrm{TiO}_{2}$ 平带电位移动 $-159 \mathrm{mV}$ 和 $89 \mathrm{mV}$. 前者有利于 $\mathrm{TiO}_{2}$ 价带与苯酚发生轨道重叠, 后者有利于 $\mathrm{TiO}_{2}$ 导带分别与 $\mathrm{O}_{2}$ 发生轨道重叠, 进而 加快界面电荷转移. 由于阴离子广泛存在, 该结果将有助于半导体光催化的机理研究及其环境应用. 关键词 锐钛矿 $\mathrm{TiO}_{2}$; 氟离子; 磷酸根; 吸附; 光电催化; 电荷转移
\end{abstract}

\section{Larger Adsorption Effect of Fluoride than Phosphate on Phenol Degradation over the Irradiated Anatase $\mathrm{TiO}_{2}$ and $\mathrm{Pt} / \mathrm{TiO}_{2}$}

\author{
Liu, Shengwei Zhao, Jianjun Xu, Yiming* \\ (Department of Chemistry and State Key Laboratory of Silicon Materials, Zhejiang University, Hangzhou 310027)
}

\begin{abstract}
It is known that fluoride and phosphate in aqueous solution can accelerate the photocatalytic degradation of phenol over anatase or $\mathrm{P}_{2} 5 \mathrm{TiO}_{2}$. But the mechanism still remains under debate. In this work, an anion-free anatase $\mathrm{TiO}_{2}$ is prepared, followed by deposition with $0.52 \mathrm{wt} \% \mathrm{Pt}\left(\mathrm{Pt} / \mathrm{TiO}_{2}\right)$. Reaction was performed in aqueous solution at initial $\mathrm{pH} 5.2$, where $99 \%$ of anions were in the form of $\mathrm{F}^{-}$or $\mathrm{H}_{2} \mathrm{PO}_{4}^{-}$. On the addition of $0.1 \sim 30 \mathrm{mmol} / \mathrm{L}$ anions, the rate constants of phenol degradation $\left(k_{\mathrm{obs}}\right)$ were all increased, confirming the positive effect of fluoride and phosphate, respectively. Interestingly, there was a linear relationship between the increase of $k_{\mathrm{obs}}$ and the amounts of anion adsorption, the slope of which became larger in the order of fluoride $>$ phosphate, and $\mathrm{Pt} / \mathrm{TiO}_{2}>\mathrm{TiO}_{2}$. These observations indicate that the positive effect of anions originates from the adsorbed anions on solid, and that fluoride was more active than phosphate. A (photo)electrochemical measurement showed that fluoride and phosphate were negative and positive, respectively, to $\mathrm{O}_{2}$ reduction, but they were all beneficial to phenol oxidation. Furthermore, in the presence of fluoride and phosphate, the flat band potentials of $\mathrm{TiO}_{2}$ were shifted by -159 and $89 \mathrm{mV}$, respectively. The former favors orbital overlapping of phenol with $\mathrm{TiO}_{2}$ valence band, and the latter favors orbital overlapping of $\mathrm{O}_{2}$ with $\mathrm{TiO}_{2}$ conduction band, all of which promotes the interfacial charge transfers. Since inorganic anions are widely present, this result would benefit the mechanism study of a semiconductor photocatalyis and its application. As a reference, pure anatase was prepared from the hydrolysis of tetrabutyl titanate, followed by calcination in air at $400{ }^{\circ} \mathrm{C}$ for $2 \mathrm{~h}$. The solid was then deposited with Pt, produced in situ from the photocatalytic reduction of $\mathrm{H}_{2} \mathrm{PtCl}_{6}$ in the presence of methanol. Solid was characterized with X-ray diffraction, $\mathrm{N}_{2}$ adsorption, Raman, and $\mathrm{X}$-ray photoelectron spectroscopy. After Pt deposition, anatase phase remained unchanged, but the solid pores were blocked by a mixture of $\mathrm{Pt}$ and $\mathrm{PtO}_{2}$. Photoreactions were performed at room temperature under UV light at wavelengths equal to and longer than $320 \mathrm{~nm}$. Organic compounds and inorganic anions were quantitatively analyzed with a high performance liquid and ionic chromatography, respectively. (Photo)electrochemical measurement was performed in a three-electrode compartment, where a Pt gauze was used as counter electrode, and a $\mathrm{AgCl} / \mathrm{Ag}$ as reference electrode.

Keywords anatase $\mathrm{TiO}_{2}$; fluoride; phosphate; adsorption; photoelectrocatalysis; charge transfer
\end{abstract}

\section{1 引言}

半导体光催化降解有机污染物被认为是治理环境 污染的重要方法之一. 人们已研究过多种半导体环境光
催化剂 ${ }^{[1 \sim 4]}$, 但在众多半导体中, $\mathrm{TiO}_{2}$ 因其高效、廉价和 稳定等优点而备受青睐 ${ }^{[5,6]}$. 根据半导体能带理论, 当入 射光的能量大于或等于半导体的禁带宽度时, 半导体的

*E-mail: xuym@zju.edu.cn; Tel.: 0571-87952410

Received January 4, 2019; published March 5, 2019.

Supporting information for this article is available free of charge via the Internet at http://sioc-journal.cn.

Project supported by the Funds for Creative Research Group of NSFC (No. 21621005).

项目受 NSFC 创新群体基金(No. 21621005)资助. 
电子就会从价带跃迁到导带, 从而分别在导带生成自由 电子 $\left(e_{\mathrm{cb}}{ }^{-}\right)$, 在价带留下自由空穴 $\left(h_{\mathrm{vb}}{ }^{+}\right)$. 然后, 这些载流 子迁移到固体表面, 进而与特定分子发生化学反应. 例 如, 锐钛矿型 $\mathrm{TiO}_{2}$ 的 $e_{\mathrm{cb}}{ }^{-}$能将表面 $\mathrm{O}_{2}$ 还原成 $\mathrm{O}_{2}^{-*}$, 相应 的 $h_{\mathrm{vb}}{ }^{+}$或将表面有机物直接氧化, 或先将表面 $\mathrm{OH}^{-} / \mathrm{H}_{2} \mathrm{O}$ 氧化, 生成表面・ $\mathrm{OH}_{\text {surf }}$, 而后再将有机物氧化. 在该过 程中, $\mathrm{TiO}_{2}$ 起到光催化剂的作用, 而本身不变. 但是, $\mathrm{TiO}_{2}$ 光生载流子寿命短, 易复合, 从而导致有机物降解 的量子效率普遍较低. 因此, 加快界面电荷转移尤为重 要. 例如, 贵金属 $\mathrm{Pt}$ 能催化 $\mathrm{O}_{2}$ 被 $e_{\mathrm{cb}}{ }^{-}$还原, 延长 $h_{\mathrm{vb}}{ }^{+}$寿 命, 从而提高有机物被 $h_{\mathrm{vb}}{ }^{+}$氧化的量子效率 ${ }^{[7]}$.

已有文献报道, 许多无机阴离子能加快 $\mathrm{TiO}_{2}$ 光催 化降解水中有机物, 并提出了多种可能的机理, 其中关 于氟离子和磷酸根的研究较多 ${ }^{[8 \sim 17]}$. Minero 等 ${ }^{[12,13]}$ 认为, $\mathrm{F}^{-}$取代表面 $\mathrm{OH}^{-}$, 迫使 $\mathrm{TiO}_{2}$ 的 $h_{\mathrm{vb}}{ }^{+}$氧化溶剂 $\mathrm{H}_{2} \mathrm{O}$, 进而 产生游离态 ${ }^{\circ} \mathrm{OH}_{\text {free }}$. 因为 ${ }^{\cdot} \mathrm{OH}_{\text {free }}$ 的反应活性高于 ${ }^{\circ} \mathrm{OH}_{\text {surf, }}$, 这加快了苯酚的降解. Choi 等 ${ }^{[14,15]}$ 指出, $\mathrm{F}^{-}$的作用与有

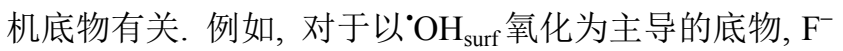
呈现正作用; 而对于以 $h_{\mathrm{vb}}{ }^{+}$氧化为主导的底物, $\mathrm{F}^{-}$呈现 负作用. Zhao 等 ${ }^{[17]}$ 认为, $\mathrm{F}^{-} 、 \mathrm{PO}_{4}{ }^{3-}$ 和 $\mathrm{SO}_{4}{ }^{2-}$ 在 $\mathrm{TiO}_{2}$ 表面 形成负电场, 再通过静电作用将 $h_{\mathrm{vb}}{ }^{+}$吸引至固体表面, 进而抑制 $h_{\mathrm{vb}}{ }^{+}$与 $e_{\mathrm{cb}}{ }^{-}$复合, 提高 $h_{\mathrm{vb}}{ }^{+}$氧化 $\mathrm{H}_{2} \mathrm{O} / \mathrm{OH}^{-}$和有 机物的效率. 我们 ${ }^{[18 ~ 20]}$ 发现, 在板钛矿 $\mathrm{TiO}_{2}$ 光催化降 解苯酚和二氯苯酚过程中, $\mathrm{F}^{-} 、 \mathrm{PO}_{4}{ }^{3-} 、 \mathrm{SO}_{4}{ }^{2-}$ 和 $\mathrm{B}_{4} \mathrm{O}_{7}{ }^{2-}$ 都 具有正作用; 在锐钛矿 $\mathrm{TiO}_{2}$ 降解苯酚过程中, $\mathrm{Pt}$ 与 $\mathrm{B}_{4} \mathrm{O}_{7}{ }^{2-}$ 和 $\mathrm{CO}_{3}{ }^{2-}$ 存在协同作用, 并提出了可能的机理. $\mathrm{Pt}$ 作为电子转移助催化剂, 加速 $\mathrm{O}_{2}$ 被 $e_{\mathrm{cb}}$-还原; 而 $\mathrm{B}_{4} \mathrm{O}_{7}{ }^{2-}$ 和 $\mathrm{CO}_{3}{ }^{2-}$ 则作为空穴转移助催化剂, 它们首先被 $h_{\mathrm{vb}}{ }^{+}$氧 化, 产生的阴离子自由基再氧化苯酚, 进而阴离子复原. 但是, 以上的大多数研究均采用商品 P25 为光催化剂. 该 $\mathrm{TiO}_{2}$ 来源于 $\mathrm{TiCl}_{4}$ 水解，大约含有 $80 \%$ 锐钛矿和 $20 \%$ 金红石, 也含有氯离子等其它杂质, 进而或多或少影响 $\mathrm{TiO}_{2}$ 光催化过程. 再者, 以上的多种可能机理都基于无 机阴离子吸附, 但缺乏定量研究. 因此, 需要采用不含 无机阴离子的 $\mathrm{TiO}_{2}$ 为光催化剂, 定量地研究不同无机 阴离子的吸附量与有机物降解速率之间的关系.

以钛酸四正丁酯为前驱体, 采用溶胶凝胶法, 在不 含无机阴离子的介质中, 制备出纯锐钛矿 $\mathrm{TiO}_{2}$ (记为 $\mathrm{sAT}$ ), 并且通过光沉积法, 得到 $\mathrm{Pt}$ 负载的 $\mathrm{TiO}_{2}$ (记为 $\mathrm{Pt} / \mathrm{sAT}$ ). 然后, 在 $\mathrm{pH} 5.2$ 的水悬浮液中, 测定 $\mathrm{F}^{-}$和 $\mathrm{PO}_{4}{ }^{3-}$ 的吸附量 $(q)$ 和苯酚的光催化降解速率 $\left(k_{\mathrm{obs}}\right)$. 我们发现, $k_{\mathrm{obs}}$ 与 $q$ 之间均呈线性关系, 但斜率互不相同. 为此, 采 用光电化学方法, 进一步研究了界面电荷转移, 并提出 关于阴离子正效应的可能反应机理.

\section{2 结果与讨论}

\section{1 材料的表征}

图 1 是固体表征的实验图. sAT 和 $\mathrm{Pt} / \mathrm{sAT}$ 的 X-射线
衍射(XRD)图谱基本相同(图 1A), 都符合锐钛矿 $\mathrm{TiO}_{2}$ 的 标准图(PDF \#71-1166). 根据 101 晶面衍射峰和 Scherrer 公式, 计算出晶粒尺寸 $\left(d_{\mathrm{XRD}}\right), \mathrm{sAT}$ 和 $\mathrm{Pt} / \mathrm{sAT}$ 的 $d_{\mathrm{XRD}}$ 分别 等于 13.3 和 $13.2 \mathrm{~nm}$. 因 $\mathrm{Pt}$ 含量低(1 wt $\%), \mathrm{Pt} / \mathrm{sAT}$ 未出 现金属 $\mathrm{Pt}$ 的衍射峰. 在 $\mathrm{TiO}_{2} / \mathrm{FTO}$ 的 XRD 图中, 既出现 锐钛矿的衍射峰, 也出现 $\mathrm{SnO}_{2}$ 的衍射峰 (PDF \#41-1445). 前者说明以胶体法得到的 $\mathrm{TiO}_{2}$ 也是锐钛矿, 后者是因为 FTO 玻璃片含有 $\mathrm{SnO}_{2}$ 涂层所致.

为了进一步确认 $\mathrm{sAT}$ 的纯度, 图 $1 \mathrm{~B}$ 表示 $\mathrm{sAT}$ 的 拉曼光谱. 在 $100 \sim 800 \mathrm{~cm}^{-1}$ 范围内, 出现 153、405、 512 和 $635 \mathrm{~cm}^{-1}$ 四个明显的振动峰, 分别归属锐钛矿 的 $E_{g} 、 B_{1 g} 、 A_{1 g}$ 和 $E_{g}$ 振动 ${ }^{[21,22]}$. 此外, 未出现金红石 $\left(442 \mathrm{~cm}^{-1}\right)$ 和板钛矿 $\left(541 \mathrm{~cm}^{-1}\right)$ 的特征振动峰. 因此, 本 文获得的 sAT 是纯晶相锐钛矿 $\mathrm{TiO}_{2}$.

在 Pt 4f 的光电子能谱中(图 1C), 出现 70.4、73.8 和 $73.8 \mathrm{eV}$ 三个信号峰. 前两个峰分别归结于金属 $\mathrm{Pt}$ 的 $4 \mathrm{f}_{7 / 2}$ 和 $4 \mathrm{f}_{5 / 2}$, 而后一个峰则属于 $\mathrm{PtO}_{2}{ }^{[23 \sim 26]}$. 这说明 $\mathrm{Pt} / \mathrm{sAT}$ 含有 $\mathrm{Pt}^{0}$ 和 $\mathrm{PtO}_{2}$. 在 $\mathrm{N}_{2}$ 吸附一脱附等温线中, 出现 一个回滞环, 表明样品具有介孔结构. 根据吸附数据, 分别计算出固体的 BET 比表面积 $\left(A_{\mathrm{sp}}\right)$ 、总孔体积 $\left(V_{\mathrm{p}}\right)$ 和 平均孔径 $\left(d_{\mathrm{p}}\right)$, 结果列于表 $\mathrm{S} 1$. 与 $\mathrm{sAT}$ 相比, $\mathrm{Pt} / \mathrm{sAT}$ 的 $A_{\mathrm{sp}}$ 和 $V_{\mathrm{p}}$ 较小, 但 $d_{\mathrm{p}}$ 较大. 扫描电镜显示, $\mathrm{Pt}$ 负载前后, 固 体形貌几乎不变, 颗粒均为球状(图 S1). 这些现象表明, $\mathrm{TiO}_{2}$ 孔道被 $\mathrm{Pt}$ 粒子填充或堵塞, 从而导致孔体积和比 表面积变小, 以及平均孔径变大.

\section{2 苯酚降解}

本文以苯酚作为模型底物, 以波长大于 $320 \mathrm{~nm}$ 的 紫外光为光源. 在这些条件下, 水中苯酚不发生光解, 它在 $\mathrm{TiO}_{2}$ 表面的吸附也可忽略不计. 因此，根据苯酚的 浓度变化, 可直接比较不同催化剂之间的光催化性能. 图 2 表示阴离子对光催化降解苯酚的影响. 随着光照时 间的增加，水中苯酚浓度呈指数性下降，符合准一级动 力学方程(图 S2). 一般认为, 当光强等一定时, $h_{\mathrm{vb}}{ }^{+}$等活 性物种的浓度衡定，有机物被氧化的速率则与其浓度成 正比. 由图可见, Pt/sAT 的光催化活性高于 sAT; 加入氟 离子或磷酸根, 都能提高 $\mathrm{sAT}$ 和 $\mathrm{Pt} / \mathrm{sAT}$ 的光催化活性. 因 $\mathrm{Pt} / \mathrm{sAT}$ 的比表面积小于 $\mathrm{sAT}$, 这表明 $\mathrm{Pt}$ 促进 $\mathrm{TiO}_{2}$ 光 催化降解有机物, 氟离子和磷酸根具有正效应.

在苯酚降解过程中，1,2-羟基苯(HQ)是主要中间产 物(图 S3). 随着光照进行, 水中 HQ 浓度增加. 时间一定 时, HQ 浓度的大小是: (1) $\mathrm{Pt} / \mathrm{sAT}>\mathrm{sAT}$; (2) $\mathrm{sAT}+\mathrm{F}^{-}>$ $\mathrm{PO}_{4}{ }^{3-}>$ 无阴离子; (3) $\mathrm{Pt} / \mathrm{sAT}+\mathrm{F}^{-}>$无阴离子 $>\mathrm{PO}_{4}{ }^{3-}$. 产物生成速率的顺序与苯酚降解速率几乎一致, 但是 HQ 的生成浓度大约是苯酚消失浓度的 $10 \%$. 这些现象 说明, HQ 等中间产物一旦生成, 它们也同时降解, 而 $\mathrm{Pt}$ 和阴离子的存在都没有显著改变苯酚的降解过程.

\section{3 阴离子吸附等温线}

图 3 表示阴离子在 $\mathrm{sAT}$ 和 $\mathrm{Pt} / \mathrm{sAT}$ 上的吸附等温线. 
随着阴离子平衡浓度 $\left(C_{\mathrm{eq}}\right)$ 增加, 阴离子的吸附量 $(q)$ 增 大, 尔后趋向极值. 这些吸附等温线均符合 Langmuir 单 分子吸附方程(图 $\mathrm{S} 4$ ), $q / q_{\mathrm{max}}=K C_{\mathrm{eq}} /\left(1+K C_{\mathrm{eq}} q\right)$, 其中 $q_{\text {max }}$ 和 $K$ 分别表示最大吸附量和吸附常数, 结果列于表 1. 与氟离子相比, 磷酸根具有较大的最大吸附量和吸 附常数. 根据多重平衡计算, 在 $\mathrm{pH} 5.20$ 的水溶液中, $99 \%$ 的氟离子和磷酸根分别以 $\mathrm{F}^{-}$和 $\mathrm{H}_{2} \mathrm{PO}_{4}{ }^{-}$存在. 相对于 $\mathrm{F}^{-}, \mathrm{H}_{2} \mathrm{PO}_{4}{ }^{-}$水合离子的半径较大, 理应具有较小 $q_{\max }$. 这 说明水合阴离子的电荷量和尺寸都不是主要的影响因 素. 有研究表明, 氟离子吸附是取代 $\mathrm{TiO}_{2}$ 表面 $\mathrm{OH}^{-}$基
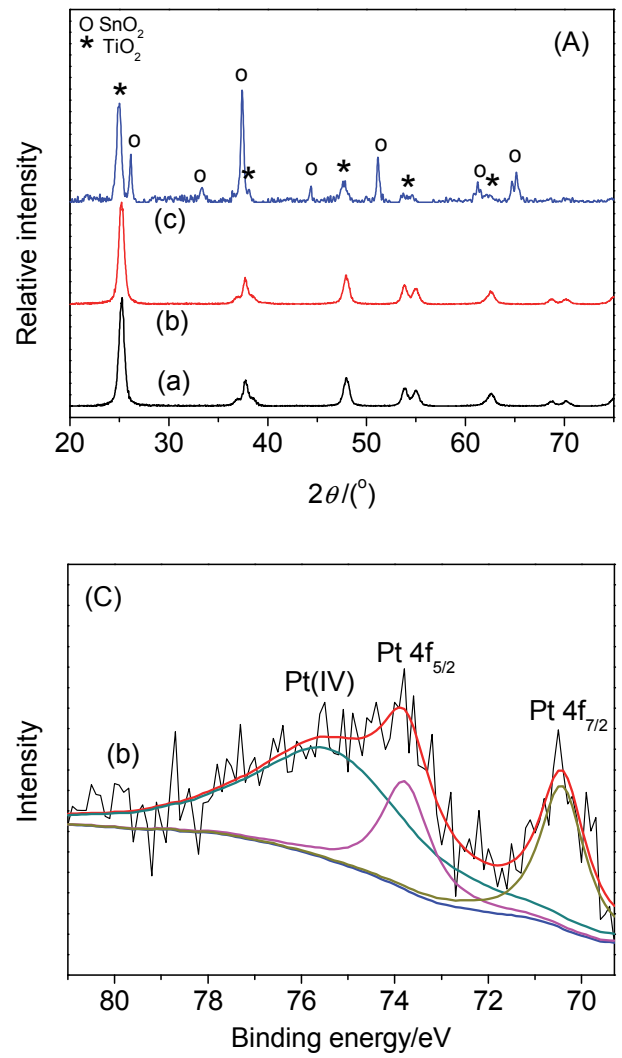

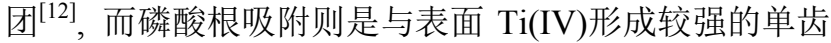
或双齿配合物 ${ }^{[16]}$. 因此，氟离子和磷酸根的吸附模式不 同造成了它们的吸附参数差异. 此外, Pt/sAT 的最大吸 附量和吸附常数都大于 $\mathrm{sAT}$, 这表明 $\mathrm{Pt}$ 与阴离子之间存 在较强的相互作用.

\section{4 阴离子浓度和表面吸附的影响}

图 4A 和 $4 \mathrm{~B}$ 表示阴离子总浓度 $\left(C_{0}\right)$ 对苯酚降解速率 提高倍数 $\left(k_{\mathrm{x}} / k_{0}\right)$ 的影响, 其中 $k_{\mathrm{x}}$ 和 $k_{0}$ 分别代表在有无阴 离子存在下苯酚降解的速率常数. 随着 $C_{0}$ 增大, $k_{\mathrm{x}} / k_{0}$ 数


图 1 样品 XRD (A), Raman (B), Pt 4f XPS (C) 和 $\mathrm{N}_{2}$ 吸附等温线(D)

Figure 1 XRD patterns (A), Raman spectra (B), Pt 4f spectra (C) and $\mathrm{N}_{2}$ adsorption-desorption isotherms (D) for the samples of (a) sAT, (b) Pt/sAT, and (c) $\mathrm{TiO}_{2} /$ FTO glass
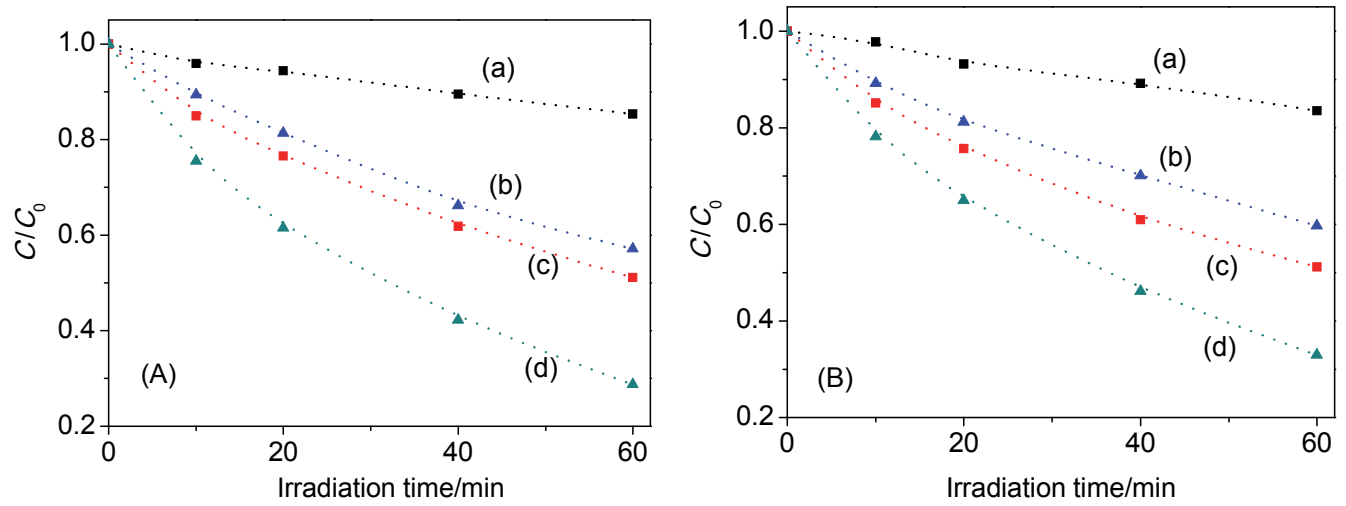

图 2 氟离子 $(\mathrm{A})$ 和磷酸根 $(\mathrm{B})$ 对光催化降解苯酚的影响

Figure 2 Effect of fluoride (A) and phosphate (B) on photocatalytic degradation of phenol. Samples were (a) sAT, (b) Pt/sAT, (c) sAT $+10 \mathrm{mmol} / \mathrm{L}$ anions, and (d) $\mathrm{Pt} / \mathrm{sAT}+10 \mathrm{mmol} / \mathrm{L}$ anions 


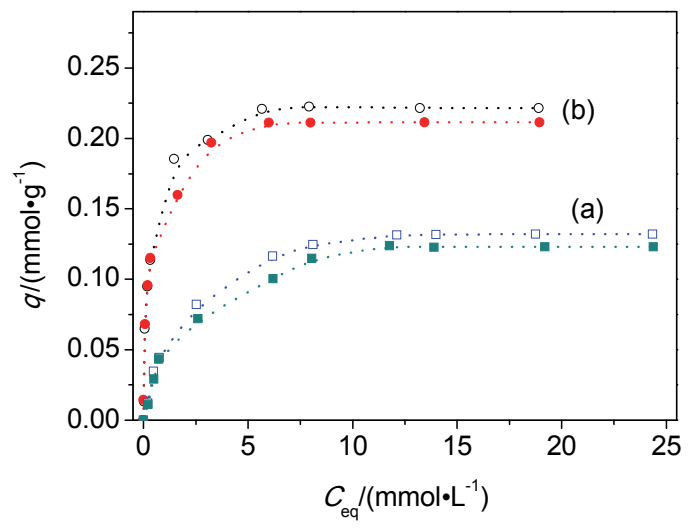

图 3 氟离子 (a) 和磷酸根 $(b)$ 的吸附等温线

Figure 3 Adsorption isotherms of fluoride (a) and phosphate (b) Samples were sAT (solid symbols), and Pt/sAT (open symbols)

值增大, 达到最大值, 然后又减小. 在浓度范围内, 氟 离子和磷酸根的 $k_{\mathrm{x}} / k_{0}$ 数值都大于 1 , 表示它们对光催化 反应都具有正效应. 相比之下, sAT 的 $k_{\mathrm{x}} / k_{0}$ 增幅和最大 值都大于 Pt/sAT. 这是因为 sAT 自身的光催化活性低于 $\mathrm{Pt} / \mathrm{sAT}$ (图 2), 从而受到阴离子的影响较大. 需要指出的 是, 在氟离子和磷酸根存在下, sAT 的光催化活性仍然 都低于 $\mathrm{Pt} / \mathrm{sAT}$ (图 S5).

对照图 3 和图 4 , 发现 $k_{\mathrm{x}} / k_{0}$ 的最大值均在 $q$ 最大值 的附近. 这意味着, $k_{\mathrm{x}} / k_{0}$ 和 $q$ 之间可能存在定量关系. 但
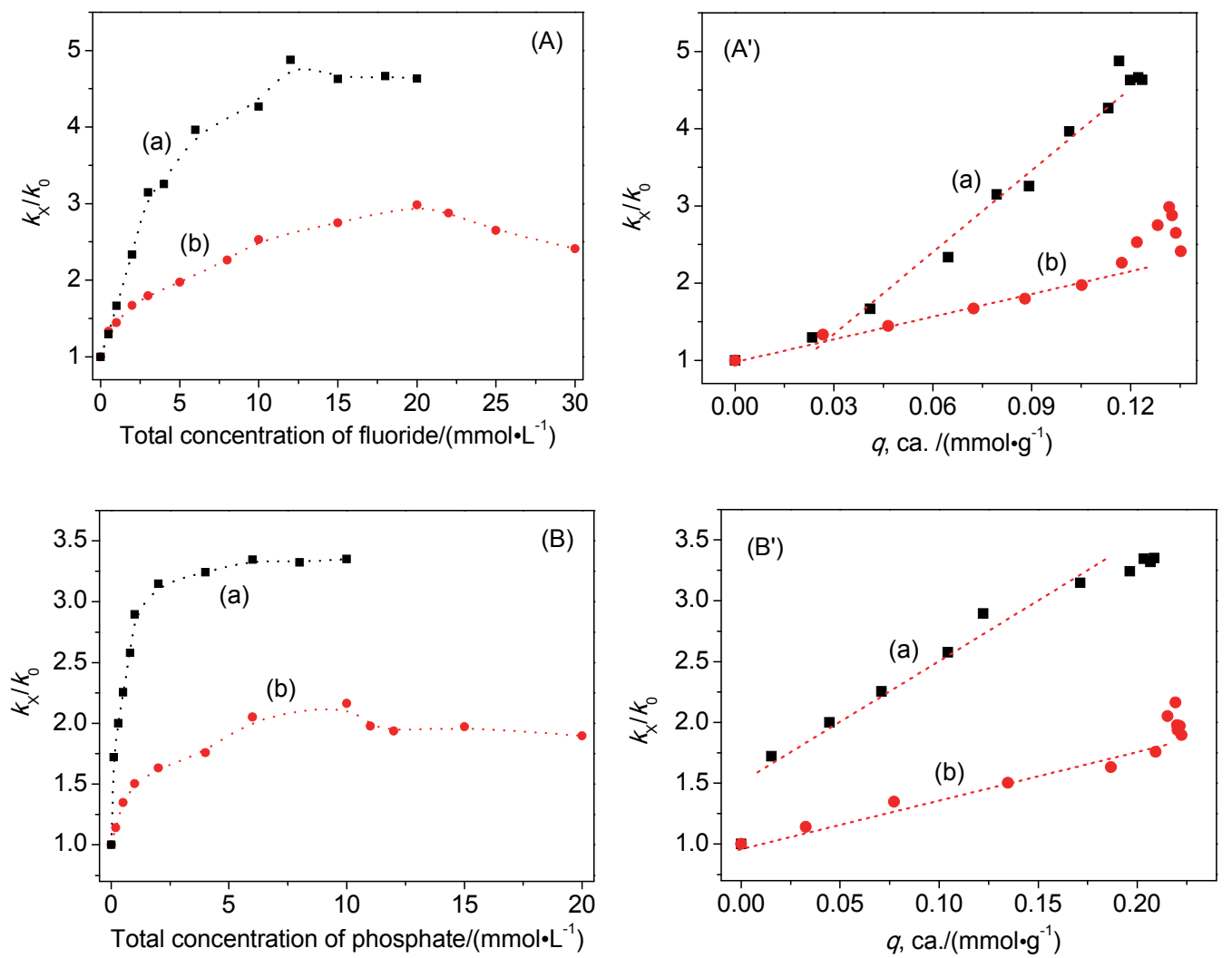

图 4 氟离子 $\left(\mathrm{A}, \mathrm{A}^{\prime}\right)$ 和磷酸根 $\left(\mathrm{B}, \mathrm{B}^{\prime}\right)$ 浓度与吸附量的影响

Figure 4 Concentration and adsorption effect of fluoride $\left(\mathrm{A}, \mathrm{A}^{\prime}\right)$ and phosphate $\left(\mathrm{B}, \mathrm{B}^{\prime}\right)$, where $k_{0}$ and $k_{\mathrm{x}}$ represent the rate constants of phenol degradation measured in absence and presence of anions, respectively. Samples were (a) sAT, and (b) Pt/sAT 
明 $\mathrm{Pt}$ 与阴离子存在某种相互作用. 在 $\mathrm{sAT}$ 和 $\mathrm{Pt} / \mathrm{sAT}$ 体 系中, 氟离子的斜率分别是磷酸根的 2.4 和 3.18 倍, 这 说明氟离子的正效应确实大于磷酸根.

\section{5 (光)电化学测试}

为了理解阴离子对界面电荷转移的影响, 我们以锐 钛矿 $\mathrm{TiO}_{2}$ 薄膜电极, 分别研究了光电化学氧化苯酚和 电化学还原氧气. 图 5 表示在 $\mathrm{N}_{2}$ 氛围下, $\mathrm{TiO}_{2}$ 薄膜电极 的线性扫描伏安(LSV)曲线. 随着外加偏压的增大, 光

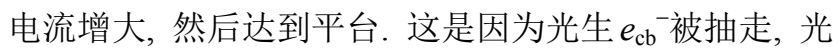
生 $h_{\mathrm{vb}}{ }^{+}$用于氧化水或者苯酚. 随着外加偏压的增大, 光 生载流子的分离效率提高. 当光生载流子的生成和复合 达到平衡时, 它们的浓度达到饱和, 相应的光电流也达 到饱和. 可以看到, 加入苯酚, 电极的光电流显著增大. 这说明苯酚比水更容易被 $h_{\mathrm{vb}}{ }^{+}$氧化, 从而提高光生载流 子的分离效率, 增大了电极的光电流. 加入磷酸根, 水 氧化和苯酚氧化的光电流都上升. 这说明, 磷酸根能促 进 $h_{\mathrm{vb}}{ }^{+}$转移, 提高 $e_{\mathrm{cb}}{ }^{-}$和 $h_{\mathrm{vb}}{ }^{+}$的分离效率, 从而分别增大 了 $h_{\mathrm{vb}}{ }^{+}$氧化水和苯酚的光电流. 奇怪的是, 加入氟离 子, 苯酚氧化的光电流上升, 而水氧化的光电流则下 降. 有文献报道, 在 $\mathrm{pH} 3.5$ 水溶液中, 氟离子在 $\mathrm{TiO}_{2}$
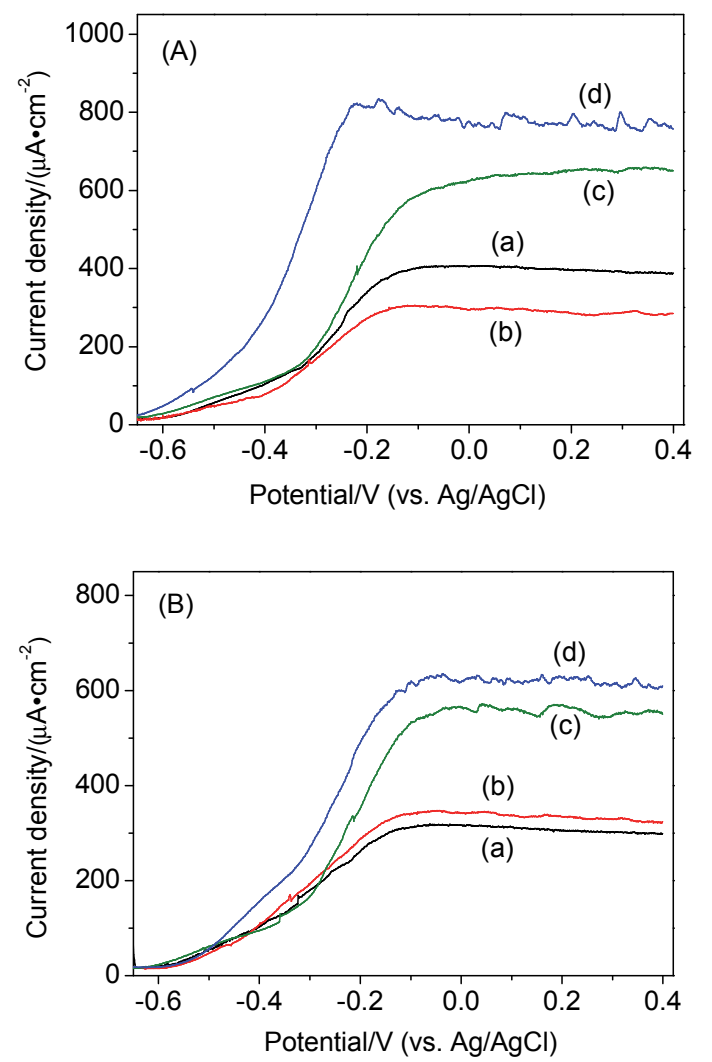

图 5 氟离子(A)和磷酸根(B)对光电氧化水和苯酚的影响

Figure 5 Photoelectrochemical oxidation of water and phenol in the presence of fluoride (A) and phosphate (B). Experiment was performed under $\mathrm{N}_{2}$ in $0.5 \mathrm{~mol} / \mathrm{L} \mathrm{NaClO}_{4}$ at $\mathrm{pH} 5.2$, (a) $\mathrm{TiO}_{2}$, (b) $\mathrm{TiO}_{2}+5 \mathrm{mmol} / \mathrm{L}$ anions, (c) $\mathrm{TiO}_{2}+0.43 \mathrm{mmol} / \mathrm{L}$ phenol, and (d) $\mathrm{TiO}_{2}+0.43 \mathrm{mmol} / \mathrm{L}$ phenol $+5 \mathrm{mmol} / \mathrm{L}$ anions
表面的吸附量最大 ${ }^{[12]}$. 为此, 在 $\mathrm{pH} 3.5$ 的 $\mathrm{NaClO}_{4}$ 水溶 液中, 测定了电极 LSV 曲线, 结果如图 S7 所示. 但 是, 氟离子和磷酸根的影响都与图 5 的结果相同. 以 上实验现象表明, 氟离子和磷酸根都能促进光生空穴 氧化苯酚; 而对于光生空穴氧化水，氟离子和磷酸根 则分别起到抑制和促进的作用.

图 6 表示在暗条件下, $\mathrm{TiO}_{2}$ 薄膜电极的 LSV 曲线图. 外加电位越负, 电极电流越大. 相比之下, $\mathrm{O}_{2}$ 气氛下的 电流远大于 $\mathrm{N}_{2}$ 气氛下的电流. 这表明电极电流主要来 源于氧气还原，而不是质子还原. 加入氟离子后，电极 电流减小，并且起始电位向负电位方向移。这说明氟离 子吸附于 $\mathrm{TiO}_{2}$ 表面, 不利于 $\mathrm{O}_{2}$ 吸附和还原. 但是, 加入 磷酸根, 随着偏压变负, 氧还原电流则是先增大后减小. 有研究表明, 磷酸根与 $\mathrm{TiO}_{2}$ 表面的 $\mathrm{Ti}(\mathrm{VI})$ 形成配合物 $(\mathrm{Ti}-\mathrm{O}-\mathrm{P}-\mathrm{OH})$, 间接吸附 $\mathrm{O}_{2}$, 从而加快 $\mathrm{O}_{2}$ 还原 ${ }^{[27]}$. 但是, 随着 $\mathrm{O}_{2}$ 还原的进行, 质子浓度下降 $\left(\mathrm{O}_{2}+4 \mathrm{H}^{+}=\right.$ $\left.2 \mathrm{H}_{2} \mathrm{O}\right)$. 因起始 $\mathrm{pH}$ 等于 5.2 , 磷酸 $\mathrm{p} K_{\mathrm{a} 2}$ 等于 $7.2, \mathrm{TiO}_{2}$ 表 面等电点 $\mathrm{pH}$ 等于 6.5 , 这导致 $\mathrm{H}_{2} \mathrm{PO}_{4}{ }^{-}$百分数下降, $\mathrm{HPO}_{4}{ }^{2-}$ 百分数上升, $\mathrm{TiO}_{2}$ 表面负电荷量增多, 磷酸根与 $\mathrm{TiO}_{2}$ 作用减弱, $\mathrm{Ti}-\mathrm{O}-\mathrm{P}-\mathrm{OH}$ 表面浓度下降, 从而不 利于 $\mathrm{O}_{2}$ 吸附和还原.
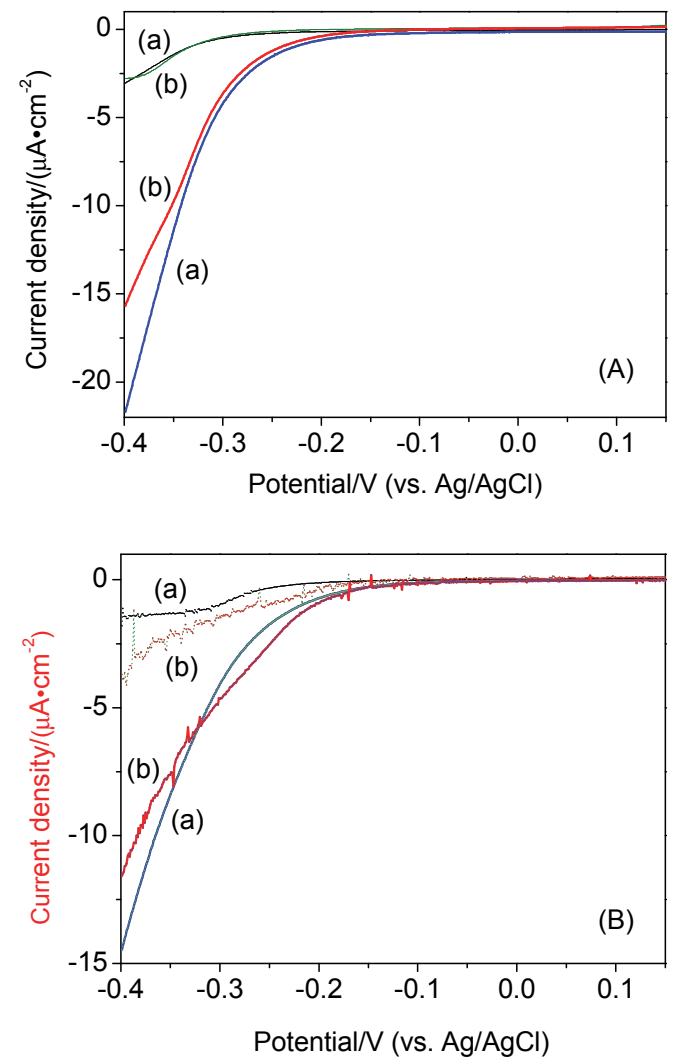

图 6 氟离子(A)和磷酸根(B)对电化学还原 $\mathrm{O}_{2}$ 的影响

Figure 6 Effect of fluoride (A) and phosphate (B) on electrochemical reduction of $\mathrm{O}_{2}$

Experiment was performed over a film electrode of (a) $\mathrm{TiO}_{2}$, and (b) $\mathrm{TiO}_{2}+5$ $\mathrm{mmol} / \mathrm{L}$ anions, in $0.5 \mathrm{~mol} / \mathrm{L} \mathrm{NaClO}_{4}$ at $\mathrm{pH} 5.2$ under $\mathrm{N}_{2}$ (dotted lines) or $\mathrm{O}_{2}$ (solid lines) 
在 $0.5 \mathrm{~mol} / \mathrm{L} \mathrm{NaClO}_{4}$ 溶液中, 还考察了阴离子对 $\mathrm{TiO}_{2}$ 薄膜电极平带电位 $\left(E_{\mathrm{fb}}\right)$ 的影响, 相应的 Mott-Schottky 曲线列于图 S8. 当氟离子浓度等于 $0 、 2.5$ 、 10 和 $20 \mathrm{mmol} / \mathrm{L}$ 时, $E_{\mathrm{fb}}$ 数值分别等于 -389 、 -469 、 -514 和 $-548 \mathrm{mV}$; 当磷酸根浓度等于 $0 、 2 、 7$ 和 15 $\mathrm{mmol} / \mathrm{L}$ 时, $E_{\mathrm{fb}}$ 数值分别等于 $-454 、-387 、-371$ 和 $-368 \mathrm{mV}$. 也就是说, 加入 $20 \mathrm{mmol} / \mathrm{L}$ 氟离子, $E_{\mathrm{fb}}$ 负移 $159 \mathrm{mV}$; 而加入 $15 \mathrm{mmol} / \mathrm{L}$ 磷酸根, $E_{\mathrm{fb}}$ 则正移 $86 \mathrm{mV}$. 这些变化趋势均与文献报道一致 ${ }^{[15,28,29]}$.

\section{6 可能机理}

锐钛矿 $\mathrm{TiO}_{2}$ 是 $\mathrm{n}$-型半导体, 其导带边电位 $\left(E_{\mathrm{CB}}\right)$ 略 接近 $E_{\mathrm{fb}}$. 在 $\mathrm{pH} 0$ 水溶液中, 锐钛矿的 $E_{\mathrm{CB}}$ 和价带边电位 $\left(E_{\mathrm{VB}}\right)$ 分别等于 -0.12 和 $3.08 \mathrm{~V}$ vs. NHE, 而 $\mathrm{O}_{2}$ 和苯酚的 单电子氧化还原电位分别为 -0.05 和 $1.43 \mathrm{~V}$ vs. NHE. 因此, $\mathrm{O}_{2}$ 能被 $\mathrm{TiO}_{2}\left(e_{\mathrm{cb}}{ }^{-}\right)$生成 $\mathrm{HO}_{2}{ }^{-}$, 而 $\mathrm{TiO}_{2}\left(h_{\mathrm{vb}}{ }^{+}\right)$既能将 苯酚氧化成苯酚阳离子自由基, 也能将水氧化成 $\mathrm{O}_{2}$ (1.23 V vs. NHE). 但是, 在 $\mathrm{pH} 3.5$ 或 $\mathrm{pH} 5.2$ 水溶液中, 苯酚却比 $\mathrm{H}_{2} \mathrm{O}$ 易被 $h_{\mathrm{vb}}{ }^{+}$氧化. 这可能是苯酚和 $\mathrm{H}_{2} \mathrm{O}$ 氧化 分别是单电子和多电子过程的缘故.

但是, 这些热力学数据不易解释氟离子和磷酸根的 影响及其差别. 一般说来, $E_{\mathrm{fb}}$ 负移时, $E_{\mathrm{CB}}$ 和 $E_{\mathrm{VB}}$ 也负移. 此时, $e_{\mathrm{cb}}$ 还原 $\mathrm{O}_{2}$ 的驱动力增强, $h_{\mathrm{vb}}{ }^{+}$与苯酚之间的轨道 重叠增大. 同理, $E_{\mathrm{fb}}$ 正移时, $E_{\mathrm{CB}}$ 和 $E_{\mathrm{VB}}$ 也正移. 此时, $e_{\mathrm{cb}}{ }^{-}$与 $\mathrm{O}_{2}$ 之间的轨道重叠增大, $h_{\mathrm{vb}}{ }^{+}$氧化苯酚的驱动力 增强. 这些变化都有利于界面电荷转移, 但各自的贡献 不尽相同. 因氟离子的 $E_{\mathrm{fb}}$ 负移较大 $(159 \mathrm{mV})$, 它主要使 $h_{\mathrm{vb}}{ }^{+}$更与苯酚发生轨道重叠, 从而加快空穴转移. 但是, $\mathrm{F}^{-}$取代了 $\mathrm{TiO}_{2}$ 表面 $\mathrm{OH}^{-}$, 这可能是氟离子抑制 $h_{\mathrm{vb}}{ }^{+}$氧化 水的原因. 相比之下, 磷酸根的 $E_{\mathrm{fb}}$ 正移较小 $(86 \mathrm{mV})$, 它主要增大 $e_{\mathrm{cb}}$-与 $\mathrm{O}_{2}$ 的轨道重叠, 进而加快电子转移. 因为 $\mathrm{TiO}_{2}$ 的 $e_{\mathrm{cb}}{ }^{-}$和 $h_{\mathrm{vb}}{ }^{+}$成对生成和消耗, 电子转移促进 空穴转移, 反之亦然. 因此, 氟离子和磷酸根都对 $\mathrm{TiO}_{2}$ 光催化降解苯酚具有正效应. 由于 $E_{\mathrm{fb}}$ 变化较大, 氟离 子的正效应大于磷酸根. 另一方面, 阴离子对 Pt/sAT 的 正效应大于对 $\mathrm{sAT}$ (图 $\mathrm{S} 6$ ). 这是因为 $\mathrm{Pt}$ 催化 $\mathrm{O}_{2}$ 被电子 还原, 阴离子催化苯酚被空穴氧化. 这两个过程相互促 进, 提高载流子分离效率, 进而加快光催化反应. 换句 话说, Pt 和阴离子分别扮演电子和空穴转移催化剂, 从 而对光催化具有协同作用.

\section{3 结论}

从定量的角度, 研究了氟离子和磷酸根对 $\mathrm{TiO}_{2}$ 光 催化降解苯酚的影响. 光催化实验表明, 苯酚降解反应 速率的提高倍数与阴离子的吸附量之间存在线性关系. 也就是说, 阴离子的正效应实际上是吸附于催化剂的阴 离子引起的. 有趣的是, 相同吸附量时, 氟离子的正效 应大约是磷酸根的 2.7 倍. 光电化学测试表明, 氟离子 和磷酸根分别减慢和加快 $\mathrm{O}_{2}$ 的电子还原, 但它们都能
促进苯酚的空穴氧化. Mott-Schottky 曲线表明，氟离子 和磷酸根分别使 $\mathrm{TiO}_{2}$ 的平带电位向负值和正值方向移 动. 这些都有利于界面载流子转移, 从而加快苯酚的光 催化降解. 但是, 氟离子对 $\mathrm{TiO}_{2}$ 平带电位的影响大约是 磷酸根的两倍. 因此, 相同吸附量时, 氟离子对 $\mathrm{TiO}_{2}$ 光 催化降解苯酚的正效应明显大于磷酸根. 在水环境中, 无机阴离子普遍存在, 它们或多或少影响环境光化学和 光催化, 本文现象和可能机理应具有一定的参考价值.

\section{4 实验部分}

\section{1 材料和试剂}

钛酸四正丁酯 $(\mathrm{TBT})$ 、无水乙醚、乙酸、苯酚、 $\mathrm{Na}_{3} \mathrm{PO}_{4} 、 \mathrm{NaF} 、 \mathrm{HNO}_{3} 、 \mathrm{NaClO}_{4} 、 \mathrm{HClO}_{4} 、 \mathrm{NaOH}$ 均购自 国药集团, 氯铂酸 $\left(\mathrm{H}_{2} \mathrm{PtCl}_{6}\right)$ 购自 Sigma-Aldrich 公司. 实 验溶液均采用 Milli-Q 超纯水配制, 溶液的 $\mathrm{pH}$ 则用 $\mathrm{HClO}_{4}$ 或 $\mathrm{NaOH}$ 的稀溶液来调节.

\section{2 催化剂的合成与表征}

根据文献方法 ${ }^{[30]}$ ，合成 sAT. $50 \mathrm{~mL}$ TBT 溶于 200 $\mathrm{mL}$ 乙醚, 加入 $60 \mathrm{~mL}$ 乙酸, 搅拌 $30 \mathrm{~min}$, 再逐滴加入 $75 \mathrm{~mL}$ 体积比为 $10: 5$ 的水和乙酸混合液, 出现白色浑 浊, 老化 $24 \mathrm{~h}$. 除去上层乙醚清液, 残余物 $100{ }^{\circ} \mathrm{C}$ 干燥, 研磨，去离子水充分洗涤，然后在 $400{ }^{\circ} \mathrm{C}$ 下㷽烧 $2 \mathrm{~h}$. 以 光化学沉积法 ${ }^{[31]}$, 制备 Pt/sAT. 配制 $50 \mathrm{~mL}$ 悬浮液 $(1 \mathrm{~g}$ $\mathrm{TiO}_{2} 、 5 \mathrm{~mL} \mathrm{CH}{ }_{3} \mathrm{OH}$ 和 $120 \mu \mathrm{L} \mathrm{H}_{2} \mathrm{PtCl}_{6}$ ), 用 $300 \mathrm{~W}$ 高压 录灯照射 $3 \mathrm{~h}$. 过滤, 去离子水充分洗涤, $80{ }^{\circ} \mathrm{C}$ 干燥. 经 计算, $\mathrm{Pt} / \mathrm{sAT}$ 大约含有 $0.52 \mathrm{wt} \% \mathrm{Pt}$.

采用下列仪器表征固体。日本 Rigaku 公司 D/max-2550/PCX XRD 仪, 法国 Horiba JobinYvon 公司 LabRAM HR Evolution 激光共聚拉曼光谱仪, 日本 Hitachi 公司 SU-8010 扫描电子显微镜, 美国 Micromeritics 公司 ASAP 2020M 物理吸附仪(150 ${ }^{\circ} \mathrm{C}$ 脱 气, $77 \mathrm{~K}$ 测定), 日本 Shimadzu 公司 ESCA Lab 220i-XL 光电子能谱仪(以 $\mathrm{C} 1 \mathrm{~s}$ 结合能 $284.6 \mathrm{eV}$ 校正).

\section{3 光催化和分析}

反应器为 Pyrex 玻璃, 外加 $25{ }^{\circ} \mathrm{C}$ 循环冷却水套. 光 源为上海亚明公司 $300 \mathrm{~W}$ 高压录灯, 距离反应器 $10 \mathrm{~cm}$. 以紫外辐照计, 测定得反应器表面的光强约等于 3.3 $\mathrm{mW} / \mathrm{cm}^{2}$. 除说明以外, 实验条件为: 悬浮液 $50 \mathrm{~mL}, 50$ $\mathrm{mg}$ 光催化剂, $0.43 \mathrm{mmol} / \mathrm{L}$ 苯酚, $10 \mathrm{mmol} / \mathrm{L} \mathrm{NaF}$ 或 $\mathrm{Na}_{3} \mathrm{PO}_{4}$, 用稀 $\mathrm{NaClO}_{4}$ 或 $\mathrm{NaOH}$ 溶液调节 $\mathrm{pH}$ 至 5.20. 悬 浮液超声 $5 \mathrm{~min}$, 搅拌 $30 \mathrm{~min}$, 然后紫外光照射. 每隔一 定时间, 取 $2 \mu \mathrm{mL}$ 悬浮液, $0.22 \mu \mathrm{m}$ 滤膜过滤. 以美国 Dionex 公司 P680 高效液相色谱仪, 分析滤液有机物浓 度(Apollo C18 反相柱, 含 $0.1 \%$ 乙酸的 $1: 1$ 甲醇水溶 液).

阴离子吸附实验步骤如下. $20 \mathrm{~mL}$ 悬浮液含有 100 $\mathrm{mg}$ 光催化剂和不同浓度 $\left(C_{0}\right)$ 的 $\mathrm{NaF}$ 或 $\mathrm{Na}_{3} \mathrm{PO}_{4}$, 调节至 
pH 5.2. 在避光下, 搅拌 $12 \mathrm{~h}$, 过滤. 以美国 Dionex 公司 ISC-90 离子色谱仪, 测定滤液中阴离子浓度. 再根据浓 度差, 计算催化剂的阴离子吸附量.

\section{4 电极制备及光电化学测试}

按文献方法, 合成 $\mathrm{TiO}_{2}$ 胶体 ${ }^{[32 \sim 34]} .25 \mathrm{~mL}$ TBT 与 $5.5 \mathrm{~g}$ 乙酸混合, 注入到 $120 \mathrm{~mL} 0.1 \mathrm{~mol} / \mathrm{L} \mathrm{HNO}_{3}$ 溶液. 加热至 $80{ }^{\circ} \mathrm{C}$, 保持 $8 \mathrm{~h}$, 再搅拌过夜. 转入聚四氟乙烯 反应管, $220{ }^{\circ} \mathrm{C}$ 反应 $12 \mathrm{~h}$. 冷却后, 旋蒸法浓缩, 质量比 达到约 $11 \%$, 置于棕色瓶内待用. 以 doctor-blade 法制备 薄膜电极. 取适量上述胶体，溶入 $50 \%$ 聚乙二醇 (PEG-2000). 将混合胶体均匀涂覆 FTO 导电玻璃, 自然 晾干, $450{ }^{\circ} \mathrm{C}$ 炦烧 $2 \mathrm{~h}$. 玻璃片切成小块, 用环氧树脂密 封, 使 $\mathrm{TiO}_{2}$ 电极暴露面积等于 $1 \mathrm{~cm} \times 1 \mathrm{~cm}$.

采用上海辰华 CHI660E 电化学工作站, 测试 $\mathrm{TiO}_{2}$ 薄膜工作电极的 LSV 曲线. $\mathrm{Pt}$ 网电极为对电极, $\mathrm{Ag} / \mathrm{AgCl}$ 电极为参比电极, 支持电解质为 $0.5 \mathrm{~mol} / \mathrm{L} \mathrm{NaClO}_{4}$ 溶液, 扫描速率为 $10 \mathrm{mV} / \mathrm{s}$. 为了避免电极厚度等物理参数的 影响, 每一组实验只用同一个电极. 电化学还原氧气的 步骤如下: 在避光条件下, 通氮气 $30 \mathrm{~min}$, 测定 LSV 曲 线. 然后, 通空气 $30 \mathrm{~min}$, 再测定 LSV 曲线. 光电化学 氧化水和苯酚的实验均在 $\mathrm{N}_{2}$ 气氛下进行, 光源是北京 畅拓 $500 \mathrm{~W}$ 氙灯. 光照前, 通氮气 $30 \mathrm{~min}$. 然后, 依次 测定 $\mathrm{LSV}$ 曲线: $\mathrm{TiO}_{2} \rightarrow \mathrm{TiO}_{2}+$ 阴离子 $\rightarrow \mathrm{TiO}_{2}+$ 苯酚 $\rightarrow \mathrm{TiO}_{2}+$ 苯酚 + 阴离子. 电极被再次使用前, 先用 $\mathrm{NaOH}$ 稀溶液洗涤, 再用超纯水洗涤, 以去除上一实验 电极上的残留物. 通过 Mott-Schottky 曲线, 测定电极的 平带电位. 先测量交流阻抗谱, 获得虚部 $Z_{\mathrm{i}}$ 、相位 $P$ 和 电容 $C=-1 /\left(2 \pi Z_{\mathrm{i}} P\right)$. 以 $C^{-2}$ 对电位 $E$ 作图, 再对直线部 分拟合, 直线与电位轴的交点即为平带电位. 为了考察 阴离子浓度的影响, 实验以同一工作电极, 阴离子浓度 从低到高, 依次测定交流阻抗谱.

\section{References}

[1] Wu, J. J.; Ji, Z. Y.; Shen, X. P.; Miao, X. L.; Xu, K. Q. Acta Chim. Sinica 2017, 75, 1207. (吴佳佳, 季振源, 沈小平, 缪绪立, 徐克 强, 化学学报, 2017, 75, 1207.)

[2] Du, P. J.; Su, T. M.; Luo, X.; Zhou, X. T.; Qin, Z. Z.; Ji, H. B.; Chen,
J. H. Chin. J. Chem. 2018, 36, 538.

[3] Chai, Y. Y.; Liu, Q. Q.; Zhang, L.; Ren, J.; Dai, W. L. Chin. J. Chem. 2017, 35, 173.

[4] Zhang, F. L.; Duan, F.; Ding, Z. G.; Chen, M. Q. Chin. J. Chem. 2017, 35, 226.

[5] Hoffmann, M.; Martin, S.; Choi, W.; W. Bahnemann, D. W. Chem. Rev. 1995, 95, 69.

[6] Tachikawa, T.; Fujitsuka, M.; Majima, T. J. Phys. Chem. C 2007, $111,5259$.

[7] Zielińska-Jurek, A.; Zaleska, A. Catal. Today 2014, 230, 104

[8] Chen, K. T.; Lu, C. S.; Chang, T. H.; Lai, Y. Y.; Chang, T. H.; Wu, C. W.; Chen, C. C. J. Hazard. Mater. 2010, 174, 598

[9] Chiang, K.; Amal, R.; Tran, T. J. Mol. Catal. A: Chem. 2003, 193, 285.

[10] Kumar, A.; Mathur, N. J. Colloid Interf. Sci. 2006, 300, 244.

[11] Lv, K. L.; Li, X. F.; Deng, K. J.; Sun, J.; Li, X. H.; Li, M. Appl.Catal. B: Environ 2010, 95, 383.

[12] Minero, C.; Mariella, G.; Maurino, V.; Pelizzetti, E. Langmuir 2000, $16,2632$.

[13] Minero, C.; Mariella, G.; Maurino, V.; Vione, D.; Pelizzetti, E. Langmuir 2000, 16, 8964.

[14] Vohra, M. S.; Kim, S.; Choi, W. J. Photochem. Photobiol., A: Chem. 2003, 160, 55.

[15] Park, H.; Choi, W. J. Phys. Chem. B 2004, 108, 4086.

[16] Yu, J. C.; Zhang, L. Z.; Zheng, Z.; Zhao, J. C. Chem. Mater. 2003, $15,2280$.

[17] Zhao, D.; Chen, C. C.; Wang, Y. F.; Ji, H. W.; Ma, W. H.; Zang, L.; Zhao, J. C. J. Phys. Chem. C 2008, 112, 5993.

[18] Zhang, X.; Xiong, X. Q.; Xu, Y. M. RSC Adv. 2016, 6, 61830.

[19] Xiong, X. Q.; Xu, Y. M. J. Phys. Chem. C 2016, 120, 3906.

[20] Xiong, X. Q.; Zhang, X.; Xu, Y. M. J. Phys. Chem. C 2016, 120, 25689.

[21] Mathpal, M. C.; Tripathi, A. K.; Singh, M. K; Gairola, S. P.; Pandey, S. N.; Agarwal, A. Chem. Phys. Lett. 2013, 555, 182

[22] Choi, H. C.; Jung, Y. M.; Kim, S. B. Vib. Spectrosc. 2005, 37, 33.

[23] Li, F. B.; Li, X. Z. Chemosphere 2002, 48, 1103.

[24] Nie, L. H.; Yu, J. G.; Li, X. Y.; Cheng, B.; Liu, G.; Jaroniec, M. Environ. Sci. Technol. 2013, 47, 2777.

[25] Yu, J. G.; Qi, L. F.; Jaroniec, M. J. Phys. Chem. C 2010, 114, 13118.

[26] Vorontsov, A. V.; Savinov, E. N.; Zhensheng, J. J. Photochem. Photobiol., A: Chem. 1999, 125, 113.

[27] Cao, Y.; Jing, L.; Shi, X.; Luan, Y.; Durrant, J. R.; Tang, J.; Fu, H. Phys. Chem. Chem. Phys. 2012, 14, 8530.

[28] Minella, M.; Maurino, V.; Minero, C.; Pelizzetti, E. J. Nanosci. Nanotechnol. 2015, 15, 3348.

[29] Nelson, B. P.; Candal, R.; Corn, R. M.; Anderson, M. A. Langmuir 2000, 16, 6094.

[30] Li, S. F.; Ye, G. L.; Chen, G. Q. J. Phys. Chem. C 2009, 113, 4031.

[31] Kim, J.; Lee, C. W.; Choi, W. Environ. Sci. Technol. 2010, 44, 6849.

[32] Barbé, C. J.; Arendse, F.; Comte, P.; Jirousek, M.; Lenzmann, F.; Shklover, V.; Grätzel, M. J. Am. Ceram. Soc. 2005, 80, 3157.

[33] Topoglidis, E.; Lutz, T.; Willis, R. L.; Barnett, C. J.; Cass, A. E. G.; Durrant, J. R. Faraday Discuss. 2000, 116, 35

[34] Willis, R. L.; Olson, C.; O'Regan, B.; Lutz, T.; Nelson, J.; Durrant, J. R. J. Phys. Chem. B 2002, 106, 7605 . 